\title{
Simplified expressions for dynamic behavior of cylindrical shells uncoupled and coupled with liquids
}

\author{
Davidson de Oliveira França Júnior ${ }^{a *}$ (DD \\ Paulo Marcelo Vieira Ribeirob \\ Lineu José Pedroso ${ }^{a}$ \\ a Universidade de Brasília, Departamento de Engenharia Civil e Ambiental, Brasília-DF, Brasil. E-mail: davidson.francajunior@gmail.com, \\ lineu@unb.br \\ b Universidade Federal de Pernambuco. Departamento de Engenharia Civil, Recife-PE, Brasil. E-mail: paulo.vribeiro@ufpe.br \\ * Corresponding author
}

https://doi.org/10.1590/1679-78255546

\begin{abstract}
The dynamic behavior of cylindrical shells is essential in many practical applications. These include in-vacuum and coupled vibrations of structures with contained fluids. Closed-form solutions are extremely complex since they involve many terms and algebraic operations that require numerical solvers. In this work, a simplified closed-form solution for the free vibration analysis of an empty or filled with an inviscid and incompressible fluid cylindrical tank is presented. The proposed analytical method is developed for a simply supported cylindrical shell, based on an energy formulation obtained with variational calculus, and provides explicit expressions for natural frequencies, which can be easily programmed in a spreadsheet. The fluid is represented by an acoustic cavity, modeled by the wave equation, and the fluid-structure interaction is reduced to an added mass of fluid in the uncoupled shell equations of motion. A finite element model was built using ANSYS software to validate the proposed procedure. The natural frequencies and mode shapes were studied, and the results obtained are consistent with analytical, numerical and experimental results.
\end{abstract}

\section{Keywords}

Cylindrical shells, free vibration, fluid-structure interaction, analytical method.

\section{INTRODUCTION}

A thin-walled cylindrical shell is defined by a solid with curved surface and small thickness compared to its other dimensions. Structural dynamic analysis of shells is elaborate due to the occurrence of simultaneous flexural and in-plane vibrations. Many concomitant factors influence the dynamic response of a cylindrical shell, like the contained material type (fluid or solid), boundary conditions, the presence of ring support, the influence of the connection between the shell walls and the bottom and the lid, among others.

The study of free vibration in cylindrical shells received and still is a topic of active interest by many researchers. Based on the classic works of Love (1888), Donnell (1933), Flugge (1934), Mushtari (1938), Reissner (1941), Vlasov (1951), Arnold and Warburton (1953), Timoshenko and Woinowsky (1959), Sanders (1959), Naghdi and Berry (1964), many other theories were developed and improved upon to simulate the dynamic behavior of a shell. These analytical techniques differ from each other by the strains and curvatures assumed for the shell, as well as by the solution methods applied to the problem. Leissa (1973) presents the different classical theories mentioned and many simulation results.

Based on classical theories, advances were made in analytical studies towards the calculation of vibration properties of cylindrical shells. Some of these studies comprise the direct solution of the equations of movement, whereas others are based on variational methods (energy based). Examples are given by the works of Sharma and Johns (1971), Chung 
(1981), Soedel (2005), Farshindianfar and Oliazadeh (2012), Chen et al. (2013), Dai et al. (2013), Sun et al. (2013), Ataabadi et al. (2014), Ameijeiras and Godoy (2016), and Qin et al. (2017). These works admit different shell theories to simulate deformations, as well as different displacement assumptions, such as: beam modal functions, characteristic orthogonal polynomials, Chebyshev polynomials, or exact Fourier series solution. These provide efficient computational methodologies to obtain the dynamic behavior of shells with classic or arbitrary boundary conditions. However, the proposed solutions are often extensive and complex for practical design applications.

On the other hand, among the different analytical methods applied to the free vibration analysis of cylindrical shells coupled with fluids, it is worth mentioning the works of Lakis and Paidoussis (1971), Gonçalves and Batista (1987), Fernholz and Robinson (1990), Sarkar and Sonti (2009), Gonçalves et al. (2010), Mendes et al. (2014), Lopez (2014), França Júnior et al. (2017) and Ji et al. (2019). In these studies, the Fluid-Structure Interaction (FSI) is conceived by adding the fluid hydrodynamic pressures in the shell movement equations, together with the impenetrability condition at the shellliquid interface. In the above-cited works, the calculation procedure is discussed and no explicit equation that expresses the frequency of the coupled fluid-shell system is presented - i.e., computational implementation is required for problem-solving.

Although analytical solutions are quite effective, theoretical-experimental studies were carried out in order to analyze the dynamic behavior of uncoupled and coupled cylindrical shells, as can be highlighted in the works of Haroun (1983), Fortuny Gasser (1987), Pedroso et. al. (1994), Amabili and Dalpiaz (1995) and Li et al. (2018). In these studies, experimental research shows that performing the FSI by adding the virtual mass of the fluid in the movement equations of the shell is satisfactory and yields excellent results.

In an overview, it can be said that both for empty and fluid-filled shells, the literature presents elaborate and extensive analytical solutions that require computational methods to solve the governing equations, which, in most cases, do not result in simple closed-form solutions. In this sense, a simplified solution that yields good results could be applied for validation purposes and in practical design analysis.

With that in mind, this work presents an innovative, compact and simplified analytical solution to calculate the dynamic behavior of simply supported cylindrical shells, uncoupled and coupled with liquids. The vibration characteristics of (i) the uncoupled shell, (ii) the uncoupled fluid and (iii) the coupled shell-liquid system (where the virtual mass of the fluid is added in the shell equations of motion) are discussed. Simplifications in the shell strains and curvature were essential to find the closed-form solution, which provides explicit expressions for natural frequencies, that can be easily programmed in a spreadsheet.

The results obtained with the proposed procedure were compared with values obtained using the finite element method through ANSYS software, and with experimental results described by Amabili and Dalpiaz (1995). The natural frequencies and mode shapes were studied, and the results obtained are consistent with analytical, numerical and experimental results.

\section{CYLINDRICAL SHELL (STRUCTURAL DOMAIN)}

The thin-walled cylindrical shell (Figure 1 ) is defined with length $L$, average radius $R$, radial angle $\phi$ and thickness $h$. The shell is made of homogenous, isotropic and elastic material with Young modulus $E$, Poisson coefficient $\mu$ and density $\rho$. The displacement vector coordinates on the surface of the shell in the axial, circumferential and radial directions are $u, v$ and $w$, respectively.

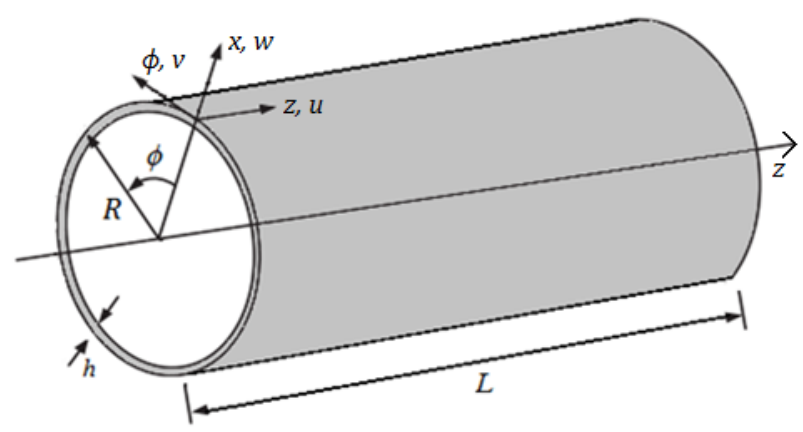

Figure 1: Schematic diagram of a thin-walled cylindrical shell.

Based on Flugge (1934) linear theory, the shell strains and curvatures are given by: 


$$
\begin{aligned}
& \varepsilon_{z}{ }^{0}=\frac{\partial u}{\partial z} ; \quad \varepsilon_{\varnothing}{ }^{0}=\frac{1}{R}\left(\frac{\partial v}{\partial \phi}+w\right) ; \quad \gamma_{z \phi}{ }^{0}=\frac{\partial v}{\partial z}+\frac{1}{R} \frac{\partial u}{\partial \phi} ; \\
& \chi_{z}=\frac{\partial^{2} w}{\partial z^{2}} ; \quad \chi_{\phi}=-\frac{1}{R^{2}}\left(\frac{\partial^{2} w}{\partial \phi^{2}}-\frac{\partial v}{\partial \phi}\right) ; \quad \chi_{z \phi}=-\frac{1}{R}\left(2 \frac{\partial^{2} w}{\partial z \partial \phi}-\frac{\partial v}{\partial z}\right)
\end{aligned}
$$

where $(0)$ is the superscript related to the mid-surface, $\varepsilon_{z}$ and $\varepsilon_{\varnothing}$ represent the membrane and transverse strains, respectively, $\gamma_{z \phi}$ denotes the shear distortions, $\chi_{z}$ and $\chi_{\phi}$ define the bending curvatures and $\chi_{z \phi}$ represents the torsional curvature. The total strains are assumed to be the sum of the membrane and flexural strains. Thus, the strain in any point of the shell is given by:

$\varepsilon_{z}=\varepsilon_{z}^{0}+x \chi_{z}$

$\varepsilon_{\varnothing}=\varepsilon_{\varnothing}^{0}+x \chi_{\phi}$

$\gamma_{z \phi}=\gamma_{z \phi}{ }^{0}+x \chi_{z \phi}$

where $x$ is the distance from a point in the shell to its mid-surface. The strains and stresses are based on Hooke's Law, and can be expressed as:

$$
\begin{gathered}
\sigma_{z}=\frac{E}{1-\mu^{2}}\left(\varepsilon_{z}+\mu \varepsilon_{\phi}\right) \\
\sigma_{\phi}=\frac{E}{1-\mu^{2}}\left(\varepsilon_{\phi}+\mu \varepsilon_{z}\right) \\
\tau_{z \phi}=\frac{E}{2\left(1-\mu^{2}\right)} \gamma_{z \phi}
\end{gathered}
$$

The equations of motion can be obtained by the Rayleigh-Ritz procedure using the Lagrange function $(\Gamma)$, given by:

$\Gamma=\mathrm{T}-\mathrm{U}$

where $\mathrm{T}$ is the total kinetic energy and $U$ is the total strain energy of the cylindrical shell. According to Brush and Almroth (1975), these expressions are given by:

$$
\begin{aligned}
& U=\frac{1}{2} \int_{-h / 2}^{h / 2} \int_{0}^{2 \pi} \int_{0}^{L}\left(\sigma_{z} \varepsilon_{z}+\sigma_{\phi} \varepsilon_{\phi}+\tau_{z \phi} \gamma_{z \phi}\right) d v \\
& T=\frac{\rho R h}{2} \int_{0}^{2 \pi} \int_{0}^{L}\left[\left(\frac{\partial u}{\partial t}\right)^{2}+\left(\frac{\partial v}{\partial t}\right)^{2}+\left(\frac{\partial w}{\partial t}\right)^{2}\right] d z d \phi
\end{aligned}
$$

where $t$ denotes time. 
The equations for $u, v$ and $w$ displacements using separation of variables and assuming harmonic vibrations of frequency $\omega$ are given by:

$$
\begin{aligned}
& u(z, \phi, t)=\bar{u}(z, \phi) e^{-j \omega t} \\
& v(z, \phi, t)=\bar{v}(z, \phi) e^{-j \omega t} \\
& w(z, \phi, t)=\bar{w}(z, \phi) e^{-j \omega t}
\end{aligned}
$$

where $e$ is the Euler's number, $\bar{u}(z, \phi), \bar{v}(z, \phi)$ and $\bar{w}(z, \phi)$ are amplitude functions and $\mathrm{j}=\sqrt{-1}$. The total strain energy and the total kinetic energy in the frequency domain are given by:

$$
\begin{aligned}
& U=\frac{E R h}{2\left(1-\mu^{2}\right)} \int_{0}^{2 \pi} \int_{0}^{L}\left(\frac{\partial u}{\partial z}\right)^{2}+\frac{1}{R^{2}}\left(\frac{\partial v}{\partial \phi}+w\right)^{2}+\frac{2 \mu}{R}\left(\frac{\partial u}{\partial z}\right)\left(\frac{\partial v}{\partial \phi}+w\right)+\frac{1}{2 R^{2}}(1-\mu)\left(\frac{\partial u}{\partial \phi}+R \frac{\partial v}{\partial z}\right)^{2} \\
& +\frac{h^{2}}{12 R^{2}}\left[\begin{array}{l}
R^{2}\left(\frac{\partial^{2} w}{\partial z^{2}}\right)^{2}+\frac{1}{R^{2}}\left(\frac{\partial^{2} w}{\partial \phi^{2}}-\frac{\partial v}{\partial \phi}\right)^{2}+2 \mu\left(\frac{\partial^{2} w}{\partial z^{2}}\right)\left(\frac{\partial^{2} w}{\partial \phi^{2}}-\frac{\partial v}{\partial \phi}\right) \\
+2(1-\mu)\left(\frac{\partial^{2} w}{\partial z \partial \phi}-\frac{1}{2} \frac{\partial v}{\partial z}\right)^{2}
\end{array}\right] d z d \phi \\
& T=\frac{\rho R h \omega^{2}}{2} \int_{0}^{2 \pi} \int_{0}^{L}[\bar{u}(z, \phi)]^{2}+[\bar{v}(z, \phi)]^{2}+[\bar{w}(z, \phi)]^{2} d z d \phi
\end{aligned}
$$

\subsection{Simplifications proposed by the authors (governing hypotheses)}

In the frequency domain, the mode shapes are given by:

$\bar{u}(z, \phi)=\mathrm{A} \frac{\partial \varphi_{m}(z)}{\partial z} \cos (n \phi)$

$\bar{v}(z, \phi)=\mathrm{B} \varphi_{m}(z) \operatorname{sen}(n \phi)$

$\bar{w}(z, \phi)=\mathrm{C} \varphi_{m}(z) \cos (n \phi)$

where $A, B$, and $C$ are amplitudes of the axial, circumferential and radial directions, respectively. In the proposed method $\varphi_{m}(z)$ defines the longitudinal mode shapes for a simply supported beam (Blevins, 1979):

$\varphi_{m}(z)=\sin \left(\frac{m \pi z}{L}\right)$

the number of longitudinal half waves is $m$ and the number of circumferential half waves is $n$.

An extensive investigation of the complete equations/formulation was done to identify possible simplifications in both the total kinetic and total strain energy expressions. This strategy is based on evaluation of relevant terms for computation of the first set of eigenvalues.

The total kinetic energy fraction associated with the axial direction was discarded, due to the displacement amplitudes in the radial and circumferential directions dominating over it. Similarly, the product between the axial and 
radial deformation, as well as the curvature in the axial direction tend to zero in the total strain energy. Under these hypotheses the simplified equations are given by:

$$
\begin{aligned}
& \tilde{U}=\frac{E R h}{2\left(1-\mu^{2}\right)} \int_{0}^{2 \pi} \int_{0}^{L}\left(\frac{\partial u}{\partial z}\right)^{2}+\frac{1}{R^{2}}\left(\frac{\partial v}{\partial \phi}+w\right)^{2}+\frac{1}{2 R^{2}}(1-\mu)\left(\frac{\partial u}{\partial \phi}+R \frac{\partial v}{\partial z}\right)^{2} \\
& +\frac{h^{2}}{12 R^{2}}\left[\frac{1}{R^{2}}\left(\frac{\partial^{2} w}{\partial \phi^{2}}-\frac{\partial v}{\partial \phi}\right)^{2}+2 \mu\left(\frac{\partial^{2} w}{\partial z^{2}}\right)\left(\frac{\partial^{2} w}{\partial \phi^{2}}-\frac{\partial v}{\partial \phi}\right)+2(1-\mu)\left(\frac{\partial^{2} w}{\partial z \partial \phi}-\frac{1}{2} \frac{\partial v}{\partial z}\right)^{2}\right] d z d \phi \\
& \tilde{T}=\frac{\rho R h \omega^{2}}{2} \int_{0}^{2 \pi} \int_{0}^{L}[\bar{v}(z, \phi)]^{2}+[\bar{w}(z, \phi)]^{2} d z d \phi
\end{aligned}
$$

After simplifying the energy terms, the Rayleigh-Ritz method applied to the Lagrange function $\tilde{\Gamma}=\tilde{T}-\tilde{U}$ is given by:

$$
\left\{\begin{array}{l}
\frac{\partial \tilde{\Gamma}}{\partial A}=0 \\
\frac{\partial \tilde{\Gamma}}{\partial B}=0 \\
\frac{\partial \tilde{\Gamma}}{\partial C}=0
\end{array}\right.
$$

Thus, a linear system of equations is obtained. These equations can be written in a compact matrix notation, with symmetrical terms, resulting in the following eigenvalue problem:

$$
\left(\boldsymbol{K}-\omega^{2} \boldsymbol{M}\right) \boldsymbol{X}=\mathbf{0} \quad ; \quad \boldsymbol{C} \boldsymbol{X}=\mathbf{0}
$$

Where $\boldsymbol{X}$ is the eigenvector. The analytical computation of $|\boldsymbol{C}|=0$ leads to the characteristic polynomial, and thus to the natural frequencies of the cylindrical shell.

The proposed procedure relies in the above-mentioned hypotheses for construction of a simplified equation for computation of the eigenvalues in (24). After some algebraic manipulations and computational assistance using MAPLE software, explicit expressions for indexes of $\boldsymbol{K}$ and $\boldsymbol{M}$ are found.

The simplified mass matrix $M$ is given by:

$$
\boldsymbol{M}=\left[\begin{array}{ccc}
m_{1,1} & 0 & 0 \\
0 & m_{2,2} & 0 \\
0 & 0 & m_{3,3}
\end{array}\right]
$$

where:

$$
\begin{aligned}
& m_{1,1}=0(26) \\
& m_{2,2}=m_{3,3}=-\frac{\rho R h \pi L}{2}
\end{aligned}
$$

The simplified stiffness matrix $\boldsymbol{K}$ is given by: 
$\boldsymbol{K}=\left[\begin{array}{lll}k_{1,1} & k_{1,2} & k_{1,3} \\ k_{2,1} & k_{2,2} & k_{2,3} \\ k_{3,1} & k_{3,2} & k_{3,3}\end{array}\right]$

where:

$k_{1,1}=\frac{E h \pi\left[-\beta_{1} \pi^{2} R^{4}+\beta_{2} L^{2} R^{2}(\mu-1)\right]}{\beta_{3} R^{3} L\left(-2 \mu^{2}+2\right)}$
$k_{1,2}=k_{2,1}=\frac{E H \pi\left[-\beta_{4} \pi L R^{3}(\mu-1)\right]}{\beta_{3} R^{3} L\left(-2 \mu^{2}+2\right)}$

$k_{1,3}=k_{3,1}=0(31)$

$k_{2,2}=\frac{E h \pi\left[\beta_{5} \pi^{2} R^{4}(\mu-1)+\beta_{6} \pi^{2} R^{2} h^{2}(\mu-1)-\beta_{7} L^{2} R^{2}-\beta_{8} L^{2} h^{2}\right]}{\beta_{3} R^{3} L\left(-2 \mu^{2}+2\right)}$

$k_{2,3}=k_{3,2}=\frac{E h \pi\left[-\beta_{9} \pi^{2} R^{2} h^{2}-\beta_{10} L^{2} R^{2}-\beta_{11} L^{2} h^{2}\right]}{\beta_{3} R^{3} L\left(-2 \mu^{2}+2\right)}$

$k_{3,3}=\frac{E h \pi\left[-\beta_{12} \pi^{2} R^{2} h^{2}-\beta_{13} L^{2} R^{2}-\beta_{14} L^{2} h^{2}\right]}{\beta_{3} R^{3} L\left(-2 \mu^{2}+2\right)}$

The coefficients $\beta_{i j}$ required for assembly of the stiffness matrix are presented in Appendix A. The characteristic polynomial is given by:

$\psi_{0} \omega^{4}+\psi_{1} \omega^{2}+\psi_{2}=0$

where:

$\psi_{0}=k_{1,1} k_{2,2} m_{3,3}$

$\psi_{1}=-k_{1,1} k_{2,2} m_{3,3}-k_{1,1} k_{3,3} m_{3,3}+k_{1,2}{ }^{2} m_{3,3}$

$\psi_{2}=k_{1,1}\left(k_{2,2} k_{3,3}-k_{2,3}^{2}\right)-k_{1,2}^{2} k_{3,3}$

Three roots of the characteristic polynomial are discarded for being imaginary, and the only real root is shown as the uncoupled cylindrical shell natural frequency equation:

$\omega_{\text {uncoupled }}=\frac{1}{2 \delta_{0}}\left[\sqrt{2} \sqrt{\delta_{0}\left(\delta_{1}+\sqrt{\delta_{2}+\delta_{3}}\right)}\right]$ 
where:

$\delta_{0}=k_{1,1} m_{2,2} m_{3,3}$

$\delta_{1}=k_{1,1} k_{2,2} m_{3,3}+k_{1,1} k_{3,3} m_{2,2}-k_{1,2}{ }^{2} m_{3,3}$

$\delta_{2}=k_{1,1}{ }^{2} k_{2,2}{ }^{2} k_{1,3}{ }^{2}-2 k_{1,1}{ }^{2} k_{2,2} k_{3,3} m_{2,2} m_{3,3}+4 k_{1,1}{ }^{2} k_{2,3}{ }^{2} m_{2,2} m_{3,3}+k_{1,1}{ }^{2} k_{3,3}{ }^{2} m_{2,2}{ }^{2}$

$\delta_{3}=-2 k_{1,1} k_{1,2}{ }^{2} k_{2,2} m_{3,3}{ }^{2}+2 k_{1,1} k_{1,2}{ }^{2} k_{3,3} m_{2,2} m_{3,3}+k_{1,2}{ }^{4} m_{3,3}{ }^{2}$

This analytical approach can be applied in practice by simply using equation (39), which can be easily programmed in a spreadsheet. Once the desired modes $(m, n)$ are chosen, the coefficients $\beta$ are selected from Table A.1 in Appendix $A$, and the natural frequency evaluated. Consequently, this procedure allows for a simple solution for the natural frequencies of an in-vacuum simply supported cylindrical shell. To the authors' knowledge no similar results are found in literature.

\section{ACOUSTIC CAVITY (FLUID DOMAIN)}

The problem is defined by a cylindrical cavity with rigid boundaries containing an homogenous fluid, where the fluid free vibrations are governed by the wave equation in cylindrical coordinates:

$\nabla^{2} P(r, \theta, z, t)=\frac{1}{c^{2}} \frac{\partial^{2} P(r, \theta, z, t)}{\partial t^{2}}$

where:

$c=\sqrt{\frac{\beta}{\rho_{f}}}$

where $\beta$ denotes the Bulk modulus, $\rho_{f}$ is the fluid density, $P(r, \theta, z, t)$ defines the acoustic pressure and $\nabla^{2}$ is the Laplacian in cylindrical coordinates.

Using separation of variables for the acoustic pressures:

$P(r, \theta, z, t)=\bar{P}(r, \theta, z) e^{-j \omega t}$

and substituting this expression in (44):

$\nabla^{2} \bar{P}(r, \theta, z)=-\frac{\omega^{2}}{c^{2}} \bar{P}(r, \theta, z)$

which leads to the Helmholtz equation for acoustic pressures of frequency $\omega$.

The following boundary conditions are applied to (47):

$\bar{P}(r, \theta, 0)=\bar{P}(r, \theta, H)=0$

$\bar{P}(r, 0, z)=\bar{P}(r, 2 \pi, z)=0$ 


$$
\left.\frac{\partial \bar{P}}{\partial r}\right|_{r=R}=0
$$

defining and open-open boundary condition in $z$, as described in the works of Gibert (1988), Pedroso (2003), Soedel (2005) and Paidoussis (2004). Solutions for fluid natural frequencies and pressure mode shapes are defined by:

$$
\begin{aligned}
& \omega_{i, m, n}=c \sqrt{\left(\frac{m \pi}{H}\right)^{2}+\left(\frac{r_{i}}{R}\right)^{2}} \\
& P_{i, m, n}(r, \theta, z)=\rho_{f} \omega^{2} A_{r} J_{n}\left(\frac{r_{i}}{R} r\right) A_{\theta} \cos (n \theta) A_{z} \operatorname{sen}\left(\frac{m \pi z}{H}\right)
\end{aligned}
$$

where $i$ is the number of nodal circles, $m$ is the number of longitudinal nodes, $n$ is the number of nodal diameters, $H$ is the acoustic cavity height, $R$ is cavity radius, $J_{n}$ is the Bessel function of first kind and order $n, r_{i}$ is the Bessel function root, $A_{R}, A_{\theta}$ and $A_{z}$ are the modal pressure amplitudes.

\section{FLUID-STRUCTURE INTERACTION}

In the shell-cylindrical cavity fluid-structure interaction (coupled problem), two boundary conditions are present: the radial pressure at the shell surface is equal to the pressure on the cavity boundary, and the radial velocity at the shell surface is equal to the velocity on the acoustic cavity surface (impenetrability condition). As a simplification, the coupled shell modes of vibration are assumed to be equal to the in-vacuum structural modes shapes.

Using the simplified analytical method of Section 2, the coupling of a simply supported cylindrical shell is done through an added fluid mass in the shell equation terms that correspond to the structure mass in the radial direction. The added mass equation ratio for a partially or filled shell is presented by Gonçalves and Batista (1987):

$$
\zeta=\left(\frac{\rho_{f}}{\rho}\right)\left(\frac{R}{h}\right)\left[\frac{H}{L}-\frac{\operatorname{sen}\left(\frac{2 m \pi H}{L}\right)}{2 m \pi}\right]\left\{\frac{J_{n}\left(r_{i}\right)}{R\left[J_{n}^{\prime}\left(r_{i}\right)\right]_{r=R}}\right\}
$$

where $\zeta$ is the virtual added mass ratio and $J_{n}^{\prime}\left(r_{i}\right)$ is the Bessel function derivative.

The added mass increases the inertial load in the shell radial direction. The shell displacements are relevant in this direction and including the added mass in this way yields more realistic results. The hydrodynamic fluid pressure is transformed into an added mass that is summed to the shell structural mass. The modified mass matrix is given by:

$$
\boldsymbol{M}=\left[\begin{array}{ccc}
m_{1,1} & 0 & 0 \\
0 & m_{2,2} & 0 \\
0 & 0 & m_{3,3}(1+\zeta)
\end{array}\right]
$$

The shell stiffness matrix is considered as the same as in (28). Once the determinant of $\boldsymbol{C}$ is evaluated and equaled to zero, the characteristic polynomial of the coupled problem is obtained. Therefore:

$$
\alpha_{0} \omega^{4}+\alpha_{1} \omega^{2}+\alpha_{2}=0
$$

where:

$$
\alpha_{0}=k_{1,1} k_{2,2}\left(m_{3,3}+\zeta\right)
$$




$$
\begin{aligned}
& \alpha_{1}=-k_{1,1} k_{2,2}\left(m_{3,3}+\zeta\right)-k_{1,1} k_{3,3} m_{2,2}+k_{1,2}{ }^{2}\left(m_{3,3}+\zeta\right) \\
& \alpha_{2}=k_{1,1}\left(k_{2,2} k_{3,3}-k_{2,3}{ }^{2}\right)-k_{1,2}{ }^{2} k_{3,3}
\end{aligned}
$$

Three roots of the characteristic polynomial are discarded for being imaginary and the real root, which represents the coupled natural frequencies, is given by:

$$
\omega_{\text {coupled }}=\frac{1}{2 \chi_{0}}\left\{\sqrt{2} \sqrt{\left[\chi_{0}\left(\chi_{1}+\sqrt{\chi_{2}+\chi_{3}}\right)\right]}\right\}
$$

with:

$$
\begin{aligned}
& \chi_{0}=k_{1,1} m_{2,2}\left(m_{3,3}+\zeta\right) \\
& \chi_{1}=k_{1,1} k_{2,2}\left(m_{3,3}+\zeta\right)+k_{1,1} k_{3,3} m_{2,2}-k_{1,2}{ }^{2}\left(m_{3,3}+\zeta\right) \\
& \chi_{2}=k_{1,1}{ }^{2} k_{2,2}{ }^{2}\left(m_{3,3}{ }^{2}+2 m_{3,3} \zeta+\zeta^{2}\right)-2 k_{1,1}{ }^{2} k_{2,2} k_{3,3} m_{2,2}\left(m_{3,3}+\zeta\right)+4 k_{1,1}{ }^{2} k_{2,3}{ }^{2} m_{2,2}\left(m_{3,3}+\zeta\right)+k_{1,1}{ }^{2} k_{3,3}{ }^{2} m_{2,2}{ }^{2} \\
& \chi_{3}=-2 k_{1,1} k_{1,2}{ }^{2} k_{2,2}\left(m_{3,3}{ }^{2}+2 m_{3,3} \zeta+\zeta^{2}\right)+2 k_{1,1} k_{1,2}{ }^{2} k_{3,3} m_{2,2}\left(m_{3,3}+\zeta\right)+k_{1,2}{ }^{4}\left(m_{3,3}{ }^{2}+2 m_{3,3} \zeta+\zeta^{2}\right)
\end{aligned}
$$

Similarly to the uncoupled problem, equation (59) can be applied in practical design problems by selecting the desired vibration modes $(m, n)$, obtaining the coefficients $\beta$ from the Table in appendix $\mathrm{A}$, evaluating the added mass for the respective shell mode with equation (53), and finally evaluating the natural frequency with equation (59). Thus, this method is a simpler approach to solve the vibration of a cylindrical shell coupled with fluids.

\section{APPLICATIONS}

\subsection{Geometry and physical parameters}

The following material properties were adopted in the models: the shell is made of steel with $E=200 G P a$, $\mu=0,29$ and $\rho=7760 \mathrm{~kg} / \mathrm{m}^{3}$. The fluid is water with $\rho_{f}=1000 \mathrm{~kg} / \mathrm{m}^{3}$ and $c=1500 \mathrm{~m} / \mathrm{s}$. The cylindrical shell is defined with $R=0,0175 \mathrm{~m}, L=0,664 \mathrm{~m}$ and $h=1,02 \mathrm{~mm}$. These dimensions were chosen based on the cylindrical tank experimentally tested by Amabili and Dalpiaz (1995).

FEM modal and harmonic analyses were performed in order to evaluate eigenvalues, eigenvectors and the coupled shell dominating displacements. A harmonic point load described by $P=100 \sin (\bar{\omega} t)$ was applied in the radial direction $(w)$, at the center of the cylindrical shell $(z=L / 2)$ at the angle $\phi=0^{\circ}$. Figure 2 shows the model geometry and the monitored analysis points. 


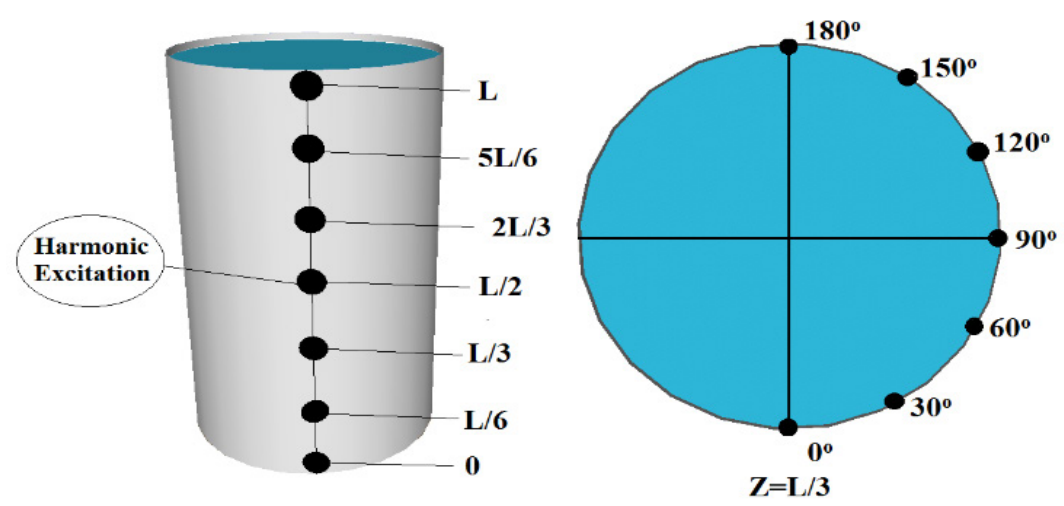

Figure 2: Geometry of the model and monitored points

\subsection{Numerical model}

The numerical analysis (Figure 3) was performed using finite elements with ANSYS software. The element SHELL63 was applied to the cylindrical shell (solid domain), whereas the element FLUID30 was applied to the acoustic cavity (fluid domain). The modules Block Lanczos and Unsymmetric were selected for the solution of eigenvalues and eigenvectors. The Full method was applied for computation of the structural response under the harmonic loads. The mesh independence analysis was performed and can be verified in França Júnior (2018). The number of elements for the final model is given by 6000 SHELL63 elements for the uncoupled shell, 13000 FLUID30 elements for the uncoupled fluid, and 21880 elements (6000 SHELL63 and 15880 FLUID30) for the coupled shell. The numerical formulation used in these simulations is based on the structure displacement and fluid pressure (U-P), given by:

$$
\left[\begin{array}{cc}
M_{s} & 0 \\
\rho F S & M_{f}
\end{array}\right]\left\{\begin{array}{l}
\ddot{U} \\
\ddot{P}
\end{array}\right\}+\left[\begin{array}{cc}
C_{s} & 0 \\
0 & C_{f}
\end{array}\right]\left\{\begin{array}{l}
\dot{U} \\
\dot{P}
\end{array}\right\}+\left[\begin{array}{cc}
K_{s} & -F S \\
0 & K_{f}
\end{array}\right]\left\{\begin{array}{c}
U \\
P
\end{array}\right\}=\left\{\begin{array}{c}
F_{E} \\
0
\end{array}\right\}
$$

where $\boldsymbol{F}_{\boldsymbol{E}}$ is a force vector, $\boldsymbol{U}$ is the structure displacement vector, $\boldsymbol{P}$ is the fluid pressure vector, $\boldsymbol{M}_{\boldsymbol{s}}$ is the structure mass matrix, $\boldsymbol{K}_{s}$ is the structure stiffness matrix, $\boldsymbol{C}_{\boldsymbol{s}}$ is the structure damping matrix, $\boldsymbol{M}_{\boldsymbol{f}}$ is the fluid mass matrix, $\boldsymbol{K}_{\boldsymbol{f}}$ is the fluid stiffness matrix, $\boldsymbol{C}_{\boldsymbol{f}}$ is the fluid damping matrix, $\boldsymbol{F S}$ is the fluid-structure coupling matrix. In the system above undamped free vibrations are established if $\boldsymbol{F}_{\boldsymbol{E}}=0, \boldsymbol{C}_{\boldsymbol{S}}=0$ and $\boldsymbol{C}_{\boldsymbol{F}}=0$.

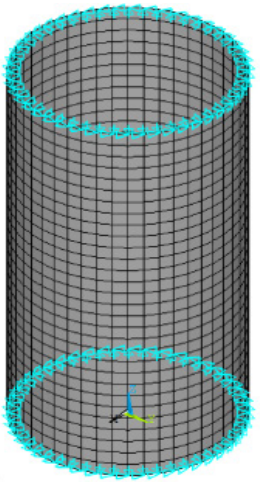

(a)

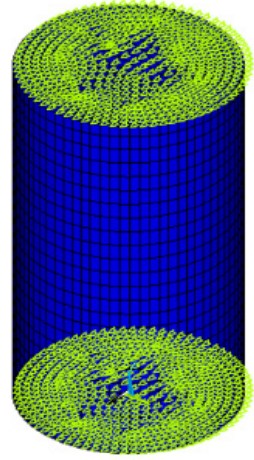

(b)

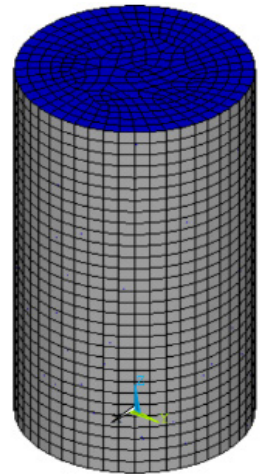

(c)

Figure 3: Numerical models: (a) uncoupled shell; (b) uncoupled fluid and (c) fluid-structure interaction.

\subsection{Results and validation}

In this section, the results obtained using the analytical method and the numerical modeling for free and forced vibrations are presented and compared. Initially, the uncoupled cylindrical shell is analyzed, then the uncoupled fluid and, finally, the coupled shell. 


\subsubsection{Cylindrical shell in free vibrations}

The first six natural frequencies obtained with the analytical technique and the numerical method are presented and compared in Table 1 . The index $N$ represents the frequency order, $m$ is the number of longitudinal half waves, and $n$ is the number of circumferential half waves. The lower natural frequencies are associated with the radial modes of vibration.

Table 1: Comparison of natural frequencies of the uncoupled cylindrical shell.

\begin{tabular}{|c|c|c|c|c|c|c|c|}
\hline$N$ & $m$ & $n$ & $\begin{array}{c}\omega_{m, n}(H z) \\
\text { Analytical }\end{array}$ & $\omega_{m, n}(H z)$ FEM & Diff. (\%) & $\begin{array}{c}\omega_{m, n}(H z) \text { Amabili } \\
\text { and Dalpiaz }\end{array}$ & Diff. (\%) \\
\hline 1 & 1 & 4 & 225,98 & 221,17 & 2,17 & 220,00 & 2,72 \\
\hline 2 & 1 & 5 & 232,80 & 230,43 & 1,03 & 230,00 & 1,22 \\
\hline 3 & 1 & 6 & 299,01 & 297,96 & 0,35 & 295,00 & 1,36 \\
\hline 4 & 1 & 3 & 322,70 & 315,92 & 2,15 & 315,00 & 2,44 \\
\hline 5 & 2 & 6 & 396,64 & 396,07 & 0,14 & 388,00 & 2,23 \\
\hline 6 & 1 & 7 & 445,62 & 441,67 & 0,89 & 440,00 & 1,28 \\
\hline
\end{tabular}

Based on Table 1, it can be noticed that the analytical and numerical results are almost the same, validating the simplified procedure. Since the difference between the results is very small for these natural frequencies, it can be concluded that the shell discretization (mesh refinement) simulates the cylindrical shell behavior for different mode shapes properly. The values obtained in the experiment by Amabili and Dalpiaz (1995) also validate the proposed formulation and the numerical method. Higher frequency results $(m=2,3,4 \ldots)$ are shown in Figure 4.

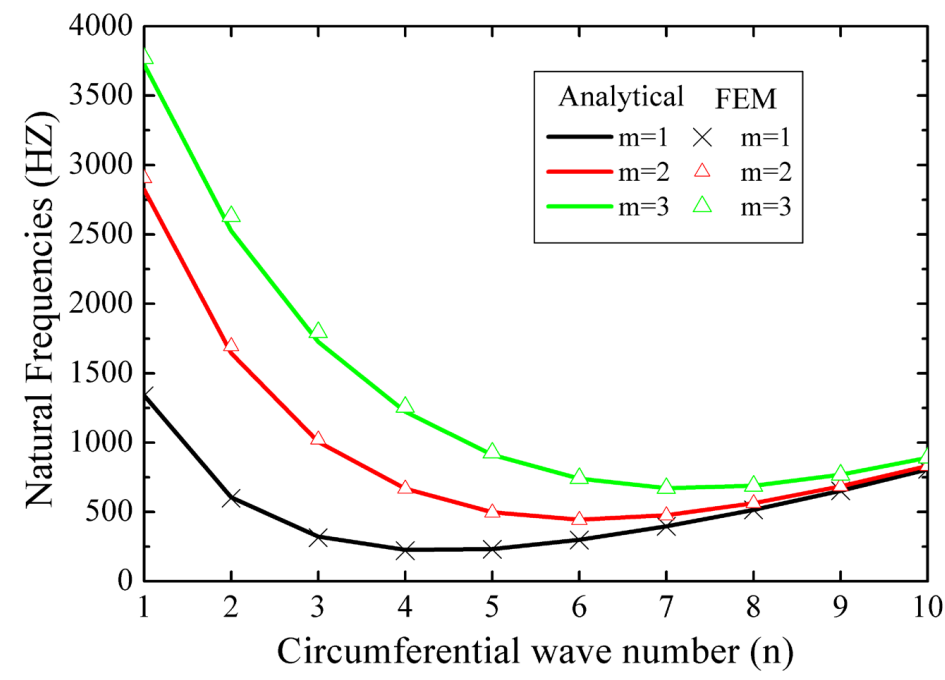

Figure 4: Natural frequencies of the uncoupled cylindrical shell.

Based on the plot in Figure 4, the cylindrical shell lower frequencies do not necessarily correspond to the lowest values of circumferential half waves, and for higher values of $n$, the natural frequencies tend to a common value. This is justified by the fact that when the shell vibrates in small circumferential modes $(n)$, the membrane longitudinal strain dominates the system total strain energy. However, as $n$ increases, the bending deformation energy becomes dominant in the system. Figure 5 shows the numerical modes of vibration obtained through modal analysis using FEM. 


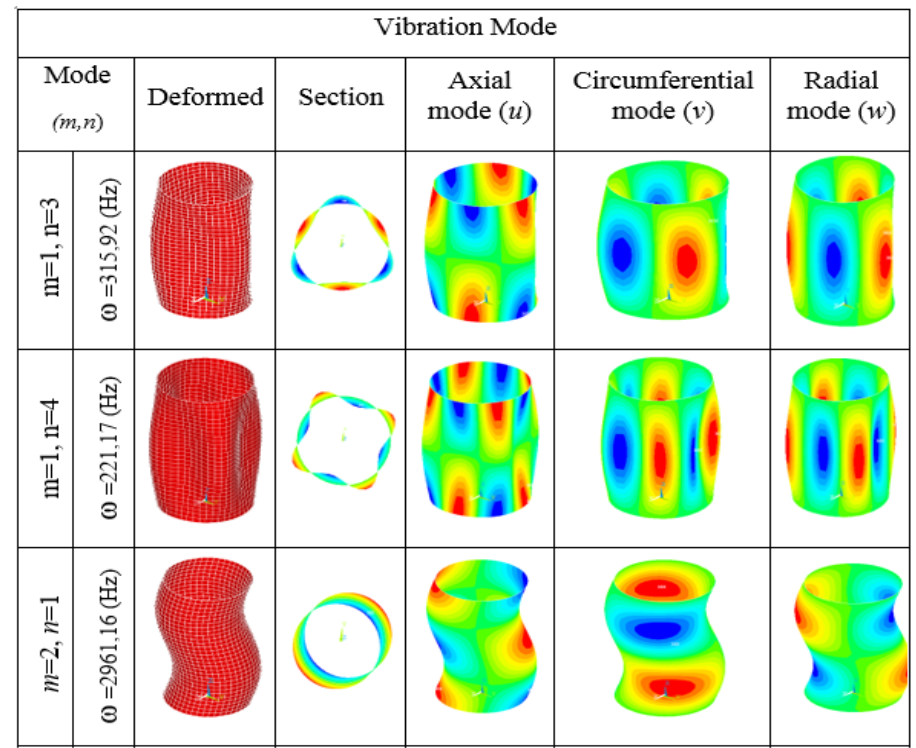

Figure 5: Natural frequencies and mode shapes of the uncoupled shell.

With the simplified formulation validated, a comparison between the results obtained using this formulation and the ones using the complete formulation is presented in Table 2. The analytical frequencies obtained with the complete formulation, including the kinetic energy (15), and strain energy (16) is defined as Analytical [A]. The analytical frequencies using the proposed method is defined as Analytical $[B]$ and is based on reduced equations (21) and (22).

Table 2: Influence of neglected terms in the formulation for natural frequencies of the cylindrical shell.

\begin{tabular}{ccccccc}
\hline$N$ & $m$ & $n$ & $\begin{array}{c}\omega_{m, n}(H z) \text { Analytical } \\
\text { [A] }\end{array}$ & $\begin{array}{c}\omega_{m, n}(H z) \text { Analytical } \\
\text { [B] }\end{array}$ & $\begin{array}{c}\omega_{m, n}(H z) \text { FEM } \\
\omega_{m, n}(H z) \text { Amabili } \\
\text { and Dalpiaz }\end{array}$ \\
\hline 1 & 1 & 4 & 221,24 & 225,98 & 221,17 & 220,00 \\
2 & 1 & 5 & 230,46 & 232,80 & 230,43 & 230,00 \\
3 & 1 & 6 & 297,99 & 299,01 & 297,96 & 295,00 \\
4 & 1 & 3 & 315,83 & 322,70 & 315,92 & 315,00 \\
5 & 2 & 6 & 396,16 & 396,64 & 396,07 & 388,00 \\
6 & 1 & 7 & 441,28 & 445,62 & 441,67 & 440,00 \\
\hline
\end{tabular}

Based on Table 2, it can be noticed that the neglected terms do not influence the results significantly. However, it is worth noting that discarding the terms of equations (15) and (16) was essential to obtain a much simpler closed-form solution for the problem.

\subsubsection{Acoustic cavity in free vibrations}

In this section, the results of the analysis of a cylindrical acoustic cavity with rigid boundaries and open-open boundary condition in $z$ are presented. Table 3 shows the analytical and numerical results of the first six natural frequencies

Table 3: Natural frequencies of the uncoupled acoustic cavity.

\begin{tabular}{cccccccc}
\hline $\boldsymbol{N}$ & $\boldsymbol{i}$ & $\boldsymbol{m}$ & $\boldsymbol{n}$ & $\boldsymbol{\omega}_{i, m, n}(\boldsymbol{H z})$ Analytical & $\boldsymbol{\omega}_{i, m, n}(\boldsymbol{H z})$ FEM & Diff. (\%) \\
\hline 1 & 0 & 1 & 0 & 1129,52 & 1130,30 & 0,07 \\
2 & 0 & 2 & 0 & 2259,04 & 2265,40 & 0,28 \\
3 & 0 & 1 & 1 & 2754,02 & 2757,20 & 0,12 \\
4 & 0 & 2 & 1 & 3378,18 & 3385,00 & 0,20 \\
5 & 0 & 3 & 0 & 3388,55 & 3409,70 & 0,62 \\
6 & 0 & 3 & 1 & 4217,95 & 4237,30 & 0,46 \\
\hline
\end{tabular}


Based on table 3, the analytical and numerical results for the acoustic cavity natural frequencies are very close to each other for the different acoustic modes. Therefore, the numerical model simulates the fluid properly. Figure 6 presents some of the acoustic cavity modes of vibration obtained through finite element method using ANSYS software.

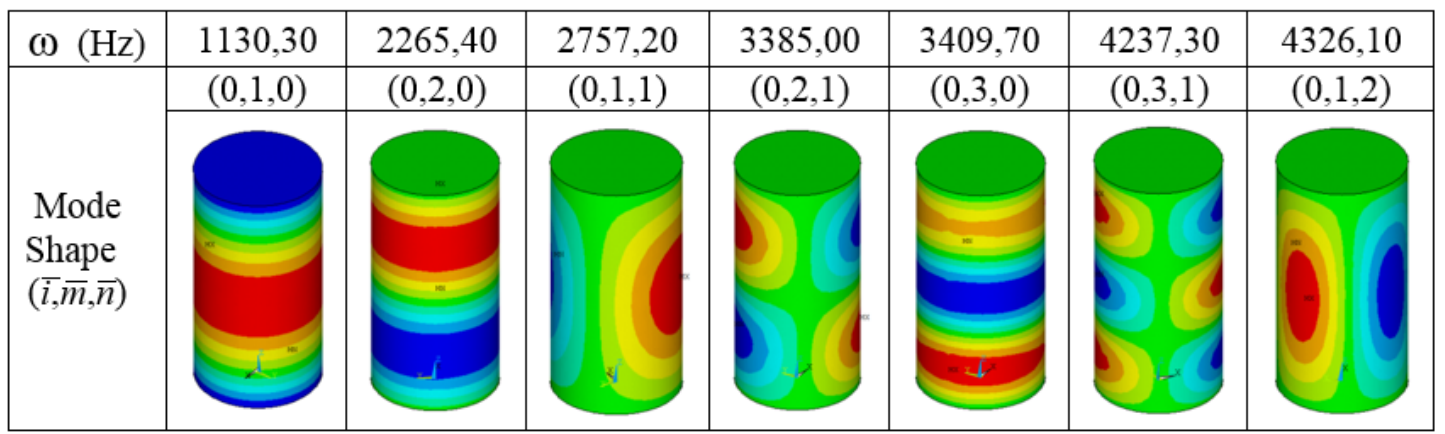

Figure 6: Natural frequencies and mode shapes of the uncoupled acoustic cavity.

The uncoupled cavity natural frequencies and modes of vibration are essential to identify the dominant modes when the structure is coupled to the fluid, as it will be shown in the next section.

\subsubsection{Fluid-structure coupled system in free and forced vibrations}

Table 4 shows the fluid-structure coupled problem natural frequencies: simply supported cylindrical shell and openopen acoustic cavity. The natural frequencies obtained through the proposed analytical methodology (adding the virtual mass term), the numerical simulation and the experimental results obtained by Amabili and Dalpiaz (1995) are compared.

Table 4: Natural frequencies of the cylindrical shell coupled with fluid.

\begin{tabular}{|c|c|c|c|c|c|c|c|c|}
\hline$N$ & $i$ & $m$ & $n$ & $\begin{array}{c}w_{i, m, n}(\boldsymbol{H z}) \\
\text { Analytical }\end{array}$ & $\omega_{i, m, n}(H z)$ FEM & Diff. (\%) & $\begin{array}{c}\omega_{i, m, n}(\boldsymbol{H z}) \\
\text { Amabilli and } \\
\text { Dalpiaz }\end{array}$ & Diff. (\%) \\
\hline 1 & 1 & 1 & 4 & 99,31 & 91,77 & 8,22 & 92,00 & 7,95 \\
\hline 2 & 1 & 1 & 5 & 112,67 & 104,91 & 7,40 & 104,00 & 8,34 \\
\hline 3 & 1 & 1 & 3 & 127,08 & 117,70 & 7,97 & 119,00 & 6,79 \\
\hline 4 & 1 & 1 & 6 & 155,46 & 147,08 & 5,70 & 147,00 & 5,76 \\
\hline 5 & 1 & 1 & 7 & 218,19 & 210,07 & 3,87 & 206,00 & 5,92 \\
\hline
\end{tabular}

The coupled cylindrical shell analytical natural frequencies presented in Table 4 are in agreement with FEM and experimental results. This validates the simplified procedure for coupled cylindrical shells in free vibration.

It is worth noting that, in the coupled problem, it was necessary to analyze the system dominating modes, because in the coupled vibration, there are: (i) structure dominating modes, where the fluid accompanies the structural displacement; (ii) acoustic cavity dominating modes, where the structure follows the fluid displacement; and (iii) mixed modes, combining results of the structure and the fluid. Thus, the coupled natural frequencies and mode shapes were compared to the uncoupled results for both the shell and the acoustic cavity.

The first twenty coupled natural frequencies are dominated by the structure with the added mass. This supports the use of the methodology presented herein. Having the formulation and modeling validated, the analysis of the coupled shell was extended to higher modes of vibration. Figure 7 presents these results. 


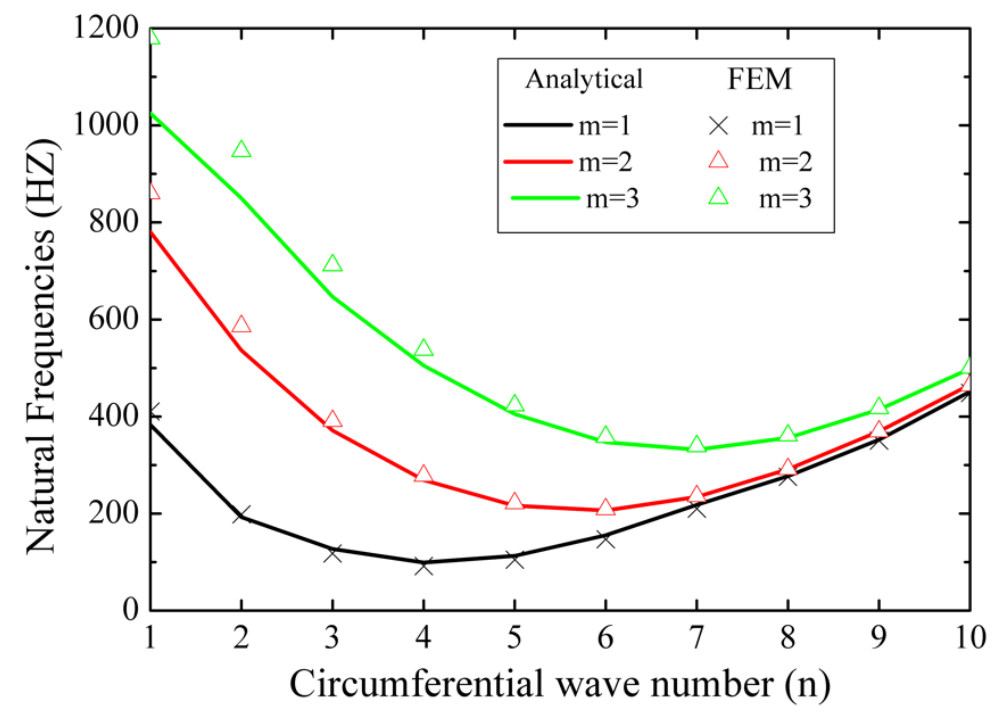

Figure 7: Natural frequencies of the cylindrical shell coupled with fluid.

Based on Figure 7 it can be noticed that the coupled shell natural frequencies behavior is similar to the uncoupled shell. According to Lakis and Paidoussis (1971), the displacement amplitudes of a simply supported shell filled with liquid are smaller if compared to the empty shell due to the fluid influence. This behavior was observed herein, showing that the fluid presence reduces the natural frequencies and reproduce the same tendencies observed in the uncoupled shell.

In the coupled low-frequency range $(3 \leq n \leq 7)$, the analytical results are in excellent agreement with the numerical values. For very high coupled frequencies ( $m=3$ and $1 \leq n \leq 3$ ), the values get closer to the fluid natural frequencies, where mixed modes of vibration start to appear. Therefore, because the cavity modes are mobilized in these cases, and the coupled formulation uses the in-vacuum structural mode shapes, the fluid added mass does not yield satisfactory results in this range of frequency, which is also expected by theory. Figure 8 shows the coupled shell mode shapes and frequencies.

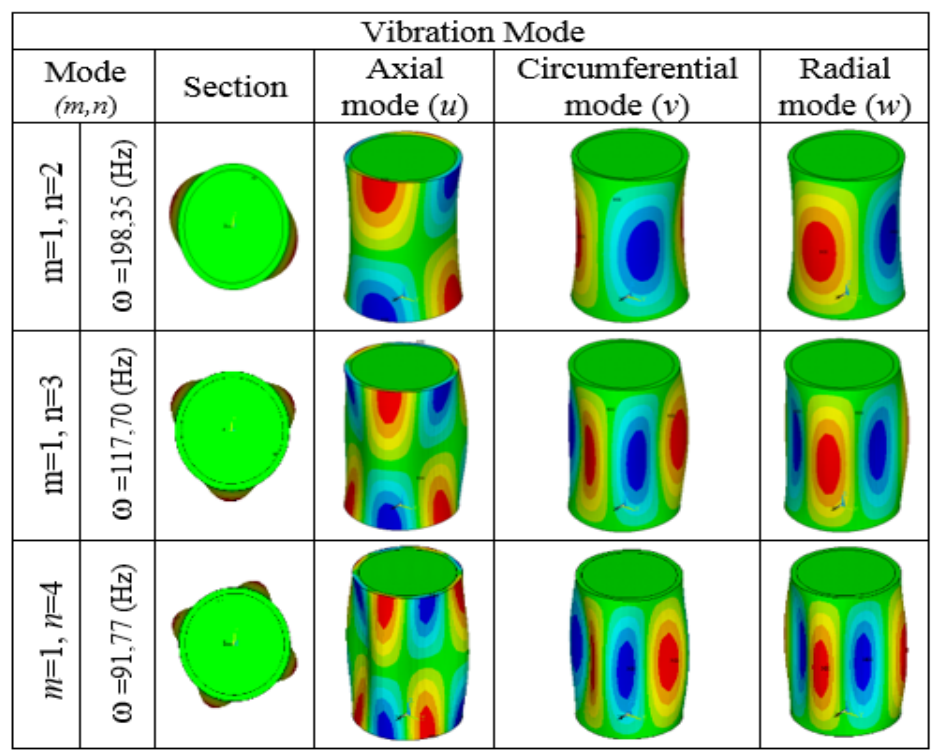

Figure 8: Natural frequencies and mode shapes of the cylindrical shell coupled with fluid.

To evaluate the dominating displacements in the fluid-filled cylindrical shell, a harmonic point load was applied in the radial direction $(w)$, at the shell center $(z=L / 2)$, and angle $\phi=0^{\circ}$. The excitation frequency $(\bar{\omega})$ varied from 0 to $200 \mathrm{~Hz}$, and the damping ratio was set to two percent $(\xi=0.02)$. Thus, it was possible to evaluate, the axial $(u)$, 
circumferential $(v)$ and radial $(w)$ displacement amplitudes as a function of the excitation frequency at the points shown in Figure 2. These results are presented in Figure 9.

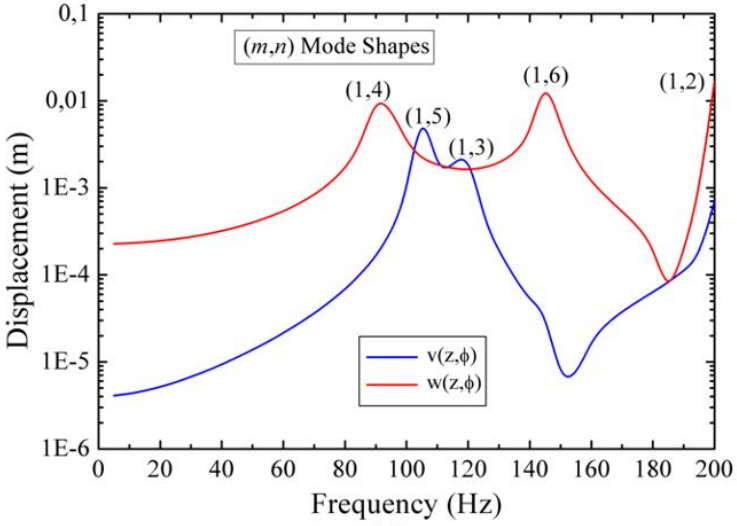

(a)

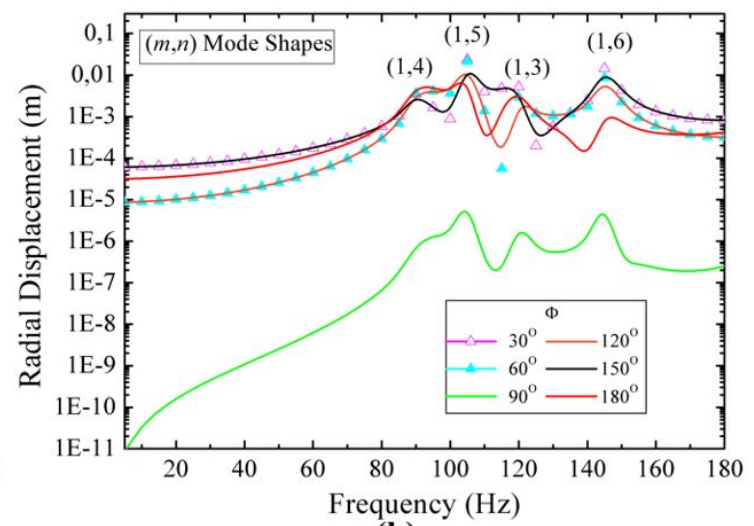

(b)

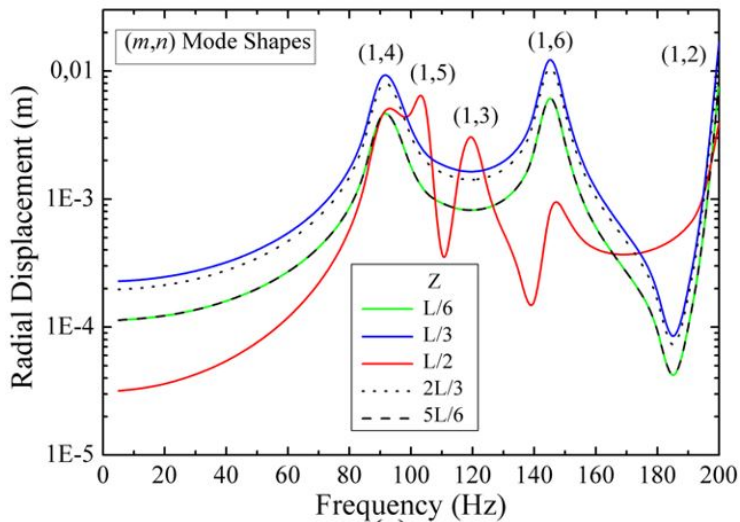

(c)

Figure 9: Frequency spectrum for a cylindrical shell coupled with fluid: (a) For $z=L / 2$ and $\varphi=180$ o; (b) For $z=L / 3$ and (c) For $\varphi=180$ o.

Figure 9 presents the radial and circumferential displacements of a single point in the shell (Fig. 9a), the radial displacement in a cross-section at different angles (Fig. 9b), and the radial displacement across the longitudinal direction (Fig. 9c). Based on the frequency spectrum presented (Fig. 9a), the peaks with more significant displacements occur at frequencies that are mainly associated with the radial and circumferential displacements. That is, the forced vibration does not mobilize the axial displacement modes, and the displacement amplitude in this direction is negligible for the first mode shapes. This is the reason why this component was not included in the plot. This fact supports the strategy of discarding the axial displacement term in the total kinetic and total strain energy equations.

\section{CONCLUSIONS}

This work addressed the aspects of fluid-structure interaction in the dynamic analysis of shells. The proposed procedure is based on an innovative, compact and simplified analytical method for computation of eigenvalues of simply supported cylindrical shells, uncoupled and coupled with liquids.

The assembly of shell mass and stiffness matrices is extremely simple and consist only of order three matrices, derived from the Rayleigh-Ritz method. Computation of the matrix indexes is straightforward and can be performed using any basic spreadsheet since explicit expressions are provided for all the involved terms. Explicit expressions for the roots of the characteristic polynomial are also available and the involved coefficients are listed in Appendix A for any given mode shape.

For fluid-filled shells, an added mass assumption (in the radial direction) proved as an extension of the uncoupled shell procedure, enabling the solution of FSI problems. This problem is extremely complicated when treated exactly, with algebraic operations that require numerical solvers. The proposed procedure is an efficient alternative since the 
simplicity of the in-vacuum shell computation is maintained, requiring only the inclusion of an added mass ratio in the last term of the main diagonal of the mass matrix.

The analysis of the acoustic cavity provided the comprehension of the uncoupled fluid dynamic behavior. This contributed to the analysis of the dominating modes in the coupled problem because the fluid and the structure vibrate at different frequency ranges.

In the fluid-structure coupled analysis, it was observed that despite influencing the system dynamic behavior, the fluid-filled shell reproduced the structure dominant mode shapes (same as the in-vacuum shell) in a significant range of analyzed frequencies. In this sense, the study of analytical techniques through the added mass criteria is significant because it applies to practical design situations in a simplified way.

Finally, it is concluded that the proposed procedure is effective whenever the governing assumptions are met, and these include (i) agreement of in-vacuum and coupled shell mode shapes; and (ii) neglecting energy terms with low representation in the Lagrange function. Under these assumptions, the procedure is reliable, with differences inferior to $10 \%$ when compared to FEM results.

\section{Acknowledgment}

This study was financed in part by the Coordenação de Aperfeiçoamento de Pessoal de Nível Superior - Brasil (CAPES) - Finance Code 001. The authors also ackowledge the support from Grupo de Dinâmica e Fluido-Estrutura (GDFE) and University of Brasilia (UnB).

Author's Contributions: Conceptualization, DO França Júnior, PMV Ribeiro and LJ Pedroso; Methodology, DO França Júnior, PMV Ribeiro and U Pedroso; Formal Analysis, DO França Júnior; Investigation, DO França Júnior and LJ Pedroso; Supervision, PMV Ribeiro and L Pedroso; Validation, DO França Júnior and $\amalg$ Pedroso; Writing - original draft, DO França Júnior; Writing - review \& editing, PMV Ribeiro and $\amalg$ Pedroso.

Editor: Rogério José Marczak.

\section{References}

Amabili, M. and Dalpiaz, G. (1995): Breathing Vibrations of a horizontal circular cylindrical tank shells, partially filled with liquid. Journal of Vibration and Acoustics, pp. 117-187.

Ameijeiras, Mariano P. and Godoy, Luis A. (2016). Simplified Analytical Approach to Evaluate the Nonlinear Dynamics of Elastic Cylindrical Shells Under Lateral Blast Loads. In: Latin American Journal of Solids and Structures 13 (2016) 1281-1298.

Arnold R. N. \& Warburton G. B. (1953): The flexural vibrations of thin cylinders. Institute Mechanical Engineering, 167 (A): $62-$ 80.

Ataabadi, Pouria Bahrami; Khedmati, Mohammad Reza and Ataabadi, Mostafa Bahrami. (2014). Free Vibration Analysis of Orthotropic Thin Cylindrical Shells with Variable Thickness by Using Spline Functions. In: Latin American Journal of Solids and Structures 12 (2014) 2099-2121.

Blevins, R. D. (1979): Formulas for Natural Frequency and Mode Shape. First Edition, Van Nostrand Renhoid Company, New York, United States.

Brush, D. O.; Almroth, B. O. (1975): Buckling of Bars, Plates, and Shells. New York: McGraw-Hill. 379 p.

Chen Y., Jin G. \& Liu Z. (2013). Free vibration analysis of circular cylindrical shell with non-uniform elastic boundary constraints. International Journal Mechanical Sciences; $74: 120-32$.

Chung H. (1981). Free vibration analysis of circular cylindrical shells. In: Journal Sound and Vibration; 74(3):331-50.

Dai, L., Yang, T., Du, J. \& Brennan, M. J., (2013). An exact series solution for the vibration analysis of cylindrical shells with arbitrary boundary conditions. International Journal Applied Acoustics, pp. 440-449.

Donnell, L. H. (1933): Stability oh thin walled tubes under torsion. Inc. NACA Report, No 479. 
Farshindianfar, A. \& Oliazadeh, P. (2012): Free vibration analysis of circular cylindrical shells: comparison of different shell theories. Journal of Mechanics and Applications, pp. 74-80.

Fernholz, C. M, Robinson, J. H. (1990): Fully-Coupled Fluid/Structure Vibration Analysis Using MSC/NASTRAN. NASA technical memorandum 102857.

Flugge, W. (1934). Statik und Dynamic der Schalen. Berlin, Julius Springer.

Fortuny Gasser, F. L. F. (1987). Vibrações de Cascas Cilíndricas Delgadas contendo Fluido. Dissertação de Mestrado, Universidade Federal do Rio de Janeiro, COOPE, Rio de Janeiro, RJ, Brasil, 138 p.

França Júnior, D. O. (2018): Estudo analítico-numérico em vibrações livres fluido-elásticas em cascas cilíndricas para diferentes condições de contorno. Dissertação de Mestrado em Estruturas e Construção Civil. Publicação E.DM-003A/18, Departamento de Engenharia Civil e Ambiental, Universidade de Brasília, Brasília, DF, 128p.

França Júnior, D. O., Pedroso, L. J. e Mendes, N. B., (2017). Estudo de Vibrações Livres Desacopladas e Acopladas FluidoEstrutura em Cascas Cilíndricas para Diferentes Condições de Contorno. CILAMCE 2018 - XXXVIII Iberian Latin American Congress on Computational Methods in Engineering, Florianópolis, SC, Brasil, 20 p.

Gibert, R. J. (1988): Vibrations des Structures. Interactions avec les fluides - Source déxcitation aléatoires. Fifth Edition, SaintGermain Paris, France, Éditions Eyrolles.

Gonçalves, P. B. and Batista, R. C., (1987): Frequency Response of Cylindrical Shells Partially Submerge dor Filled with Liquid. Journal of Sound and Vibration. DOI: 0022-460X/87/040059+12.

Gonçalves, P. B; Silva, F. M. A. and Del Prado, Z. J. G. N. (2010). Nonlinear Vibrations of Partially Fluid-Filled Cylindrical Shells. IDETC/CIE 2010 - International Design Engineering Technical Conferences \& Computers and Information in Engineering Conference, Montreal, Quebec, Canada, $8 \mathrm{p}$.

Haroun, M.; (1983). Vibration Studies and Test of Liquid Storage Tanks. In. Earthquake Engineering and Structural Dynamic. p.179-206.

Ji, M.; Inaba, K. \& Triawan F. (2019). Vibration Characteristics of Cylindrical Shells Filled with Fluid Based on First-order Shell Theory. Journal of Fluids and Structures, Vol. 85, pp. 275-291.

Lakis, A. A. \& Paidoussis, M. P. (1971): Free vibration of cylindrical shells partially filled with liquid. Journal of Sound and Vibration, pp. 1-15.

Leissa, A. W. (1973): Vibration of Shells. Technical Report NASA SP-288, Washington, D. C. pp. 425.

Li, H; Luo, H.; Wei S. \& Wen B. (2018). The Influence of Elastic Boundary on Modal Parameters of Thin Cylindrical Shell. Journal of Acoustics and Vibration, Vol. 23, pp. 93-105.

Lopez, A. A., (2014). Estudo comparativo analítico-numérico de vibrações livres e livres acopladas fluido-estrutura em cascas cilíndricas. Dissertação de Mestrado em Estruturas e Construção Civil, Departamento de Engenharia Civil e Ambiental, Universidade de Brasília, Brasília, DF, Brasil, 120p.

Love, A. E. H. (1888): The small free vibrations of a thin elastic shell. International Phil. Trans. Roy. Soc., pp. 491-549.

Mendes, N. B., Pedroso, L. J. \& Ribeiro, P. M. V., (2014): Um estudo de vibrações livres acopladas em cascas cilíndricas com anéis enrijecedores e contendo fluido. CILAMCE 2014 - XXXV Iberian Latin-American Congress on Computational Methods in Engineering, Fortaleza, CE, Brasil, 19 p.

Mushtari, K. M. (1938): On the stability of cylindrical shells subject to torsion. International Trudy Kaz. Avais, Russian.

Naghdi, P. M. \& Berry J. G. (1964). On the equations of motion of cylindrical shells. In: International Journal. Applied Mechanics. 21 (2) 160-166.

Paidoussis, M. P. (2004): Fluid-Structure Interactions - Slender Structures and Axial Flow. Volume 2, Elsevier Academic Press, McGill University, Montreal, Québec, Canada.

Pedroso, L. J. (2003): Interação Fluido-Estrutura. In: Notas de Curso e Apostila Didática, UnB-FT/ENC, Vs.3, Brasília, DF.

Pedroso, L.J.; Brito, J. L. V.; Barbosa, A. N. e Stump, R. D. (1994): Estudo Dinâmico Teórico-Experimental da Casca Cilíndrica Interna do Núcleo de um Reator Nuclear. In: 8o Simpósio Brasileiro sobre Tubulações e Vasos de Pressão e 2o Simpósio Latino Americano sobre Tubulações e Vasos de Pressão, Gramado, RS. 
Qin Z.; Chu F. \& Zu J. (2017). Free vibrations of cylindrical shells with arbitrary boundary conditions: A comparison study. In: International Journal of Mechanical Sciences; pp. 91-99.

Reissner, E. (1941): A new derivation of the equations of the deformation of elastic shells. International Am J. Math., pp. 177184

Sanders, J. L., (1959). An improved first approximation theory of thin shells. In. NASA Report.

Sarkar, A. \& Sonti, V. R. (2009). Asymptotic analysis for the coupled wavenumbers in an infinite fluid-filled flexible cylindrical shell: The beam mode. Journal of Sound and Vibration, vol. 319, pp. 646-667.

Sharma, C. B. \& Johns, D. J., (1971). Vibration characteristics of a camped-free and camped-ring-stiffened circular cylindrical shell. Inc. Journal of Sound and Vibration, pp. 459-474.

Soedel, W. (2005). Vibrations of Shells and Plates. Third Edition, Marcel Dekkler, New York, United States.

Sun W., Cao D., Han Q. (2013). Vibration studies of rotating cylindrical shells with arbitrary edges using characteristic orthogonal polynomials in the Rayleigh-Ritz method. In: Journal of Mechanical Sciences. 68 (2013) 180-189.

Timoshenko, S. \& Woinowsky, K. S., (1959). Theory of plates and shells. Second Edition, United States, McGraw-Hill.

Vlasov, V. S. (1951). Basic differential equations in general theory of elastic shells. In: National Advisory Committee for Aeronautics. NACA-TM-1241. Washington DC: US Government Printing Office. 
APPENDIX A - STIFFNESS MATRIX COEFFICIENTS

Table A.1: Stiffness Matrix Coefficients.

\begin{tabular}{|c|c|c|c|c|c|c|c|c|c|c|c|c|c|c|c|}
\hline \multicolumn{2}{|c|}{$\begin{array}{l}\text { Mode } \\
\text { Shape }\end{array}$} & \multicolumn{14}{|c|}{ Stiffness Matrix Coefficients } \\
\hline$m$ & $n$ & $\beta_{1}$ & $\beta_{2}$ & $\boldsymbol{\beta}_{3}$ & $\boldsymbol{\beta}_{4}$ & $\beta_{5}$ & $\beta_{6}$ & $\beta_{7}$ & $\beta_{8}$ & $\beta_{9}$ & $\beta_{10}$ & $\beta_{11}$ & $\beta_{12}$ & $\beta_{13}$ & $\beta_{14}$ \\
\hline 1 & 1 & 48 & 24 & 48 & 24 & 24 & 2 & 48 & 4 & 4 & 48 & 4 & 8 & 48 & 4 \\
\hline 1 & 2 & 48 & 96 & 48 & 48 & 24 & 2 & 192 & 16 & 8 & 96 & 32 & 32 & 48 & 64 \\
\hline 1 & 3 & 48 & 216 & 48 & 72 & 24 & 2 & 432 & 36 & 12 & 144 & 108 & 72 & 48 & 324 \\
\hline 1 & 4 & 48 & 384 & 48 & 96 & 24 & 2 & 768 & 64 & 16 & 192 & 256 & 128 & 48 & 1024 \\
\hline 1 & 5 & 48 & 600 & 48 & 120 & 24 & 2 & 1200 & 100 & 20 & 240 & 500 & 200 & 48 & 2500 \\
\hline 1 & 6 & 48 & 864 & 48 & 144 & 24 & 2 & 1728 & 144 & 24 & 288 & 864 & 288 & 48 & 5184 \\
\hline 1 & 7 & 48 & 1176 & 48 & 168 & 24 & 2 & 2352 & 196 & 28 & 336 & 1372 & 392 & 48 & 9604 \\
\hline 1 & 8 & 48 & 1536 & 48 & 192 & 24 & 2 & 3072 & 256 & 32 & 384 & 2048 & 512 & 48 & 16384 \\
\hline 1 & 9 & 48 & 1944 & 48 & 216 & 24 & 2 & 3888 & 324 & 36 & 432 & 2916 & 648 & 48 & 26244 \\
\hline 1 & 10 & 48 & 2400 & 48 & 240 & 24 & 2 & 4800 & 400 & 40 & 480 & 4000 & 800 & 48 & 40000 \\
\hline 2 & 1 & 96 & 12 & 24 & 24 & 48 & 4 & 24 & 2 & 8 & 24 & 2 & 16 & 24 & 2 \\
\hline 2 & 2 & 48 & 24 & 12 & 24 & 24 & 2 & 48 & 4 & 8 & 24 & 8 & 32 & 12 & 16 \\
\hline 2 & 3 & 96 & 108 & 24 & 72 & 48 & 4 & 216 & 18 & 24 & 72 & 54 & 144 & 24 & 162 \\
\hline 2 & 4 & 48 & 96 & 12 & 48 & 24 & 2 & 192 & 16 & 16 & 48 & 64 & 128 & 12 & 256 \\
\hline 2 & 5 & 96 & 300 & 24 & 120 & 48 & 4 & 600 & 50 & 40 & 120 & 250 & 400 & 24 & 1250 \\
\hline 2 & 6 & 48 & 216 & 12 & 72 & 24 & 2 & 432 & 36 & 24 & 72 & 216 & 288 & 12 & 1296 \\
\hline 2 & 7 & 96 & 588 & 24 & 168 & 48 & 4 & 1176 & 98 & 56 & 168 & 686 & 784 & 24 & 4802 \\
\hline 2 & 8 & 48 & 384 & 12 & 96 & 24 & 2 & 768 & 64 & 32 & 96 & 512 & 512 & 12 & 4096 \\
\hline 2 & 9 & 96 & 972 & 24 & 216 & 48 & 4 & 1944 & 162 & 72 & 216 & 1458 & 1296 & 24 & 13122 \\
\hline 2 & 10 & 48 & 600 & 12 & 120 & 24 & 2 & 1200 & 100 & 4 & 120 & 1000 & 800 & 12 & 10000 \\
\hline 3 & 1 & 432 & 24 & 48 & 72 & 216 & 18 & 48 & 4 & 36 & 48 & 4 & 72 & 48 & 4 \\
\hline 3 & 2 & 432 & 96 & 48 & 144 & 216 & 18 & 192 & 16 & 72 & 96 & 32 & 288 & 48 & 64 \\
\hline 3 & 3 & 144 & 72 & 16 & 72 & 72 & 6 & 144 & 12 & 36 & 48 & 36 & 216 & 16 & 108 \\
\hline 3 & 4 & 432 & 384 & 48 & 288 & 216 & 18 & 768 & 64 & 144 & 192 & 256 & 1152 & 48 & 1024 \\
\hline 3 & 5 & 432 & 600 & 48 & 360 & 216 & 18 & 1200 & 100 & 180 & 240 & 500 & 1800 & 48 & 2500 \\
\hline 3 & 6 & 144 & 288 & 16 & 144 & 72 & 6 & 576 & 48 & 72 & 96 & 288 & 864 & 16 & 1728 \\
\hline 3 & 7 & 432 & 1176 & 48 & 504 & 216 & 18 & 2352 & 196 & 252 & 336 & 1372 & 3528 & 48 & 9604 \\
\hline 3 & 8 & 432 & 1536 & 48 & 576 & 216 & 18 & 3072 & 256 & 288 & 384 & 2048 & 4608 & 48 & 16384 \\
\hline 3 & 9 & 144 & 648 & 16 & 216 & 72 & 6 & 1296 & 108 & 108 & 144 & 972 & 1944 & 16 & 8748 \\
\hline 3 & 10 & 432 & 2400 & 48 & 720 & 216 & 18 & 4800 & 400 & 360 & 480 & 4000 & 7200 & 48 & 40000 \\
\hline
\end{tabular}

